\title{
Acute stress disorder is of limited benefit in predicting post- traumatic stress disorder in people surviving traumatic injury
}

Creamer M, O'Donnell ML, Pattison P. The relationship between acute stress disorder and posttraumatic stress disorder in severely injured trauma survivors. Behav Res Therapy 2004;42:315-28.

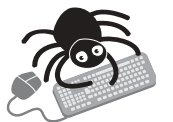

This article

contains extra text on the EBMH website

Does acute stress disorder after traumatic injury help identify people likely to develop post-traumatic stress disorder?

\section{METHODS}

-

Design: Prospective longitudinal study.

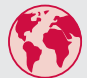

Setting: Level 1 trauma centre, Melbourne, Australia.

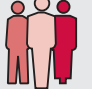

Population: 363 people aged 18-70 years who were severely injured after major trauma (mean age 36 years; $75 \%$ male). Inclusion criteria: physical injury requiring admission for at least 24 hours and free from narcotic medication for 24 hours. Exclusion criteria: moderate or severe traumatic brain injury, deliberate injury, drug dependence, psychotic illness or nonEnglish speaking.

Risk factors: Baseline assessment for acute stress disorder and post-traumatic stress disorder (PTSD) was carried out before discharge from hospital. Follow up assessment was carried out by telephone interviews conducted by a trained mental health clinician. Logistic regression analyses were used to examine whether acute stress disorder symptoms predicted PTSD. Backward elimination multiple regression was used to examine the relation between symptom severity of acute stress disorder and PTSD symptoms.

Outcomes: Acute stress disorder (CAPS-IV grouped into symptom clusters of dissociation, emotional numbing, avoidance and arousal); PTSD (CAPS-IV).

Follow up period: Three months (93\%) and 12 months (85\%).

\section{MAIN RESULTS}

At baseline, 1\% of participants were diagnosed with acute stress disorder (ASD) and 3\% of participants with PTSD. At three months, $9 \%$ of participants had PTSD, which rose to $10 \%$ at 12 months. Most people with dissociation symptoms (the core feature of ASD diagnosis) developed PTSD (see http://www.ebmentalhealth.com/ supplemental for table). However, the low sensitivity of the ASD dissociation symptom cluster produced a high percentage of falsenegative PTSD diagnoses. Reducing the number of symptoms included in the dissociation symptom cluster improved the sensitivity, but it still remained poor. In addition, many people developing PTSD did not have symptoms of dissociation during the early stages of the condition. Re-experiencing and arousal symptom clusters were significant predictors of a categorical PTSD diagnosis at three and 12 months (re-experiencing: three months, OR 4.08, 95\% CI 1.37 to 12.22 ; 12 months, OR $2.73,95 \%$ CI 1.03 to 7.22 ; arousal: three months, OR $4.9695 \%$ CI 1.52 to $16.20 ; 12$ months, OR $2.8495 \%$ CI 1.09 to 7.38$)$.

For correspondence: Mark Creamer, Department of Psychiatry, University of Melbourne, Melbourne, Australia; markcc@unimelb.edu.au

Sources of funding: the Victorian Trauma Foundation.

\section{CONCLUSIONS}

Acute stress disorder is of limited benefit in predicting post-traumatic stress disorder in people surviving traumatic injury. This is because the low sensitivity of the core dissociative symptoms of ASD results in a high number of false PTSD diagnoses. Re-experiencing and arousal symptoms may be better predictors of PTSD in this population.

\section{Commentary}

A cute stress disorder (ASD) was devised as a diagnosis to allow identification of individuals likely to develop post-traumatic stress disorder (PTSD) within one month of exposure to traumatic events. ${ }^{1}$ The aetiological model underlying ASD stresses initial dissociative processes in the development of PTSD. However, the diagnosis was adopted with little evidence of its validity. ASD diagnoses should allow us to predict efficiently who will develop PTSD, missing few cases and generating few false alarms. Creamer et al add weight to the accumulating evidence ${ }^{2-4}$ that the ASD construct does not perform as intended. Although individuals diagnosed with ASD are likely to develop PTSD, many of those who develop PTSD would not have been diagnosed with ASD.

The raising of the dissociative process to the status of "core symptom" has proven problematic. Indeed, there has been evidence for a long time that these processes are neither the sole nor primary vulnerability factors for the development of PTSD. ${ }^{1}$ The solution does not appear to be a matter of lowering the bar, as Creamer et al provide data suggesting that adjustments in the diagnostic criteria of ASD-particularly lowering the threshold for dissociative symptoms - do little to improve its predictive utility.

Creamer et al's findings regarding the predictive utility of reexperiencing and arousal symptoms are intriguing, but studies have failed to uncover consistency in the symptom clusters predicting PTSD, and Creamer et al's data do not clarify this situation. Looking to symptom clusters for prediction of PTSD may not be the most pragmatic approach and data are starting to accumulate that suggest cognitive processing factors may prove more valuable predictors. ${ }^{3}$ From the clinical standpoint, however, removing ASD as a diagnostic category and allowing PTSD to be diagnosed at any point after exposure to a traumatic stressor may make the most sense, allowing early identification of individuals with difficulties and moving diagnostic criteria away from shaky aetiological assumptions concerning the role of dissociation. Creamer et al add to the evidence that ASD does little to improve our understanding of adjustment following trauma or allow for adequate prediction of psychiatric morbidity.

Steven C Palmer, PhD Abramson Cancer Center, University of Pennsylvania, Philadelphia, PA,

1 Marshall RD, Spitzer R, Liebowitz MR. Review and critique of the new DSMIV diagnosis of acute stress disorder. Am J Psychiatry 1999;56:1677-85.

2 Bryant RA. Early predictors of posttraumatic stress disorder. Biol Psychiatry 2003;53:789-95.

3 Ginzburg K, Solomon Z, Koifman B, et al. Trajectories of posttraumatic stress disorder following myocardial infarction: a prospective study. J Clin Psychiatry 2003;64:1217-23.

4 Kassam-Adams N, Winston FK. Predicting child PTSD: The relationship between acute stress disorder and PTSD in injured children. J Am Acad Child Adolesc Psychiatry 2004;43:403-11. 